\title{
Sélection de familles de sapin de Douglas (Pseudotsuga menziesii) pour leur aptitude au bouturage : conséquences pour la diffusion de variétés améliorées
}

\author{
N Schermann, M Verger, JC Bastien \\ Station d'amélioration des arbres forestiers, Inra, 45160 Ardon, France
}

(Reçu le 3 mars 1995 ; accepté le 24 avril 1995)

\begin{abstract}
Summary - Bulk vegetative propagation of Douglas fir (Pseudotsuga menziesii) full-sib families. Vegetative propagation of elite full-sib families is one of the most promising means to obtain high genetic value material for intensive forestry. In France, selection for vegetative propagation ability would be necessary for operational production of cuttings from Douglas fir elite full-sib family seedlings, on economical and technical grounds. Potential for such a selection was appreciated on Douglas fir full-sib families from a $7 \times 7$ half-diallel (fig 1). Tip cuttings were collected on seedlings raised in greenhouse with intensive conditions for 1 year and rooted under fog in a complete block design experiment. The multiplication coefficient (Coef, number of well rooted cuttings per seedling) was broken down into three traits: number of cuttings (NB), rooting rate (Rac) and root quality (Qual). Altogether data were collected on 14573 cuttings. At the family level, all study traits (Coef, NB, Rac, Qual and height of stock plants [HT]) show high values of variability and heritability, as well as favourable correlations (tables I and II), giving rise to appreciable expected genetic gains on multiplication coefficient. Thus, in the present experiment, NB, Qual and Rac vary in a 1 to 2 ratio and Coef in a 1 to 4 ratio between extreme family values. Selecting $10 \%$ of the best families would increase the multiplication coefficient from 15 to 26 well rooted tip cuttings per seedling. Variability between full-sib families for the multiplication coefficient and its components is mostly due to general combining ability (GCA) effects (fig 2 and coefficient $R$ between full-sib families values and their values estimated by the sum of parental GCAs in table I). A path coefficient analysis on family means shows that the relationship between NB and Coef is not due to an indirect effect via the height of the stock plants. Furthermore, in this experiment, it is trait Qual that contributes the most to family variability of the multiplication coefficient (table III). For families with the highest multiplication coefficients, modification of clonal composition, due to inequal multiplication coefficients among clones within families, is of little practical importance. This means that no significant deviations are expected between the performance of an elite family tested as seedlings in progeny tests and its performance after deployment through vegetative propagation. For a practical application of these encouraging results, it is necessary to evaluate family stability for the multiplication coefficient, according to various seedling raising and cutting conditions, especially low-cost conditions.
\end{abstract}

Douglas fir / bulk vegetative propagation / genetic improvement / genetic variability / diallel 
Résumé - La propagation végétative en vrac de familles de pleins-frères d'élite de douglas est une voie prometteuse pour valoriser le progrès génétique attendu du lourd investissement consenti pour le programme français d'amélioration de l'espèce. En France, les problèmes techniques non résolus liés à l'aptitude au bouturage du douglas rendent nécessaire une sélection des familles sur ce caractère pour justifier économiquement leur multiplication à grande échelle. La possibilité d'une telle sélection a été appréciée sur 21 familles issues d'un demi-diallèle $7 \times 7$. Des boutures comportant un bourgeon terminal ont été prélevées sur des semis élevés pendant un an en serre en conditions intensives et ont été ensuite mises en enracinement sous brouillard artificiel, suivant un dispositif en blocs complets. Le coefficient de multiplication d'un pied-mère (nombre de boutures bien enracinées par pied-mère) se décompose en trois caractères : nombre de boutures prélevables, taux d'enracinement, qualité de l'enracinement. En tout, 14573 boutures ont été observées. La variabilité et l'héritabilité familiales de tous les caractères mesurés sont fortes et les liaisons entre ces caractères sont favorables. Ainsi, dans cette expérience, une sélection des $10 \%$ meilleures familles ferait passer le coefficient de multiplication de 15 à 26 . De plus, les effets d'aptitude spécifique à la combinaison des caractères liés à l'aptitude au bouturage sont faibles. Cela devrait permettre de sélectionner, sur leur valeur d'aptitude générale à la combinaison, les parents potentiels des familles de pleins-frères. Pour une application pratique, ces résultats encourageants demanderaient à être vérifiés sur d'autres itinéraires d'élevage des pieds-mères et de bouturage, notamment plus rustiques.

\section{douglas / multiplication végétative " en vrac » / amélioration génétique / variabilité génétique / diallèle}

\section{INTRODUCTION}

Le matériel génétique utilisé jusqu'à présent en France pour les reboisements en douglas vert (Pseudotsuga menziesii (Mirb) Franco var menziesii) est constitué presque exclusivement de variétés à base génétique large : variétés populations issues de graines récoltées en peuplements naturels ou artificiels et variétés synthétiques obtenues en vergers à graines.

La longueur des cycles de sélection/recombinaison chez les arbres forestiers conduit l'améliorateur à choisir à court terme la création de variétés à base génétique plus étroite comme moyen le plus rapide d'obtenir un gain génétique supplémentaire par rapport aux variétés existantes.

Chez les arbres forestiers, trois types de variétés à base génétique étroite peuvent être envisagées (Libby, 1990) :

- variétés mono- ou polyclonales,

- variétés obtenues par supplémentation pollinique en masse en verger à graines, avec des pères sélectionnés, - variétés comprenant une ou plusieurs fa- milles de pleins-frères, avec ou sans multiplication par voie végétative.

C'est la voie clonale qui, a priori, procurerait à court terme le gain génétique le plus élevé, sous réserve d'une vérification de la stabilité intra- et intersites des performances en reboisement (Kleinschmit, 1979). Pour le douglas, la faisabilité de la production de variétés clonales reste néanmoins à démontrer en raison notamment i) de la proportion importante de clones présentant une qualité d'enracinement insuffisante et ii) du vieillissement rapide des arbres sur lesquels on prélève les boutures, appelés pieds-mères, se traduisant par une baisse rapide de l'aptitude à l'enracinement et par une croissance plagiotrope des boutures (Michaud et al, 1990).

La création de variétés "familles de pleins-frères " constitue un compromis entre la voie clonale et la voie "vergers à graines ». Elle permet théoriquement d'obtenir un gain génétique au moins 1,5 fois supérieur à celui réalisable avec une variété issue de verger de clones d'élite (Schermann 1994), en conservant néanmoins une variabilité génétique à la variété qui 
devrait assurer sa stabilité dans des conditions de milieu variées. Ce supplément de gain génétique attendu provient en grande partie d'une intensité de sélection accrue. En effet, on retient en général une trentaine de génotypes au moins pour une variété « verger à graines de clones » et seulement quelques familles, à caractéristiques bien ciblées, pour une variété « familles de pleins-frères ». Ce gain supplémentaire sera d'autant plus élevé que l'on pourra isoler des familles à forts effets favorables d'aptitude spécifique à la combinaison (ASC) pour un caractère (Talbert et al, 1993), ou qui échappent à des liaisons génétiques défavorables entre caractères.

À l'heure actuelle, la mise à disposition des reboiseurs d'un matériel issu de familles de pleins-frères de douglas passe par la réalisation de croisements contrôlés en parcs à clones, suivie de la propagation par bouturage horticole, sans identification clonale, de jeunes semis issus de ces croisements (multiplication végétative « en vrac "). L'extrême juvénilité du matériel optimise l'enracinement et le retour à l'orthotropie des boutures. Dans le monde, cette méthode de création variétale connaît une

\begin{tabular}{ll|lllllll} 
& & \multicolumn{6}{c}{ Génotypes utilisés comme femelles } \\
\cline { 3 - 8 } & & 1 & 2 & 3 & 4 & 5 & 6 & 7 \\
\hline $\mathrm{c}$ & 1 & & & & & & & \\
0 & 2 & 20 & & & & & & \\
$\mathrm{~m}$ & & & & & & & \\
$\mathrm{~m}$ & 3 & 20 & 20 & & & & \\
$\mathrm{c}$ & & & & & & & \\
$\mathrm{m}$ & 4 & 20 & 18 & 20 & & & \\
$\hat{\mathrm{a}}$ & 5 & 21 & 20 & 20 & 13 & & \\
$\mathrm{l}$ & & & & & & & \\
$\mathrm{e}$ & 6 & 20 & 20 & 20 & 18 & 20 & \\
$\mathrm{~s}$ & & & & & & & \\
& 7 & 20 & 20 & 19 & 13 & 20 & 20
\end{tabular}

Fig 1. Schéma du plan de croisement diallèle réalisé. Les emplacements vides correspondent aux croisements non-inclus dans l'expérience. Pour chaque famille de plein-frères est indiqué le nombre de pieds-mères de la famille multipliés par bouturage. application à grande échelle essentiellement pour l'épicéa de Sitka en Grande-Bretagne (Fletcher, 1992) et le pin radiata en Nouvelle-Zélande (Shelbourne, 1986). Elle a aussi été essayée pour le pin maritime en France (Alazard, 1992) et est employée dans le programme d'amélioration génétique du douglas vert de la compagnie nordaméricaine Weyerhaeuser. Cette compagnie produit déjà annuellement plus d'un million de boutures issues de familles de pleins-frères d'élite (Ritchie, 1993). Les qualités physiologique et morphologique des plants obtenus sont comparables à celle de semis $1+1$ (Ritchie, 1992).

Jusqu'à présent, aucun effort n'a été consenti pour la sélection de familles de douglas à fort coefficient de multiplication (nombre moyen de boutures bien enracinées produites à partir d'un pied-mère).

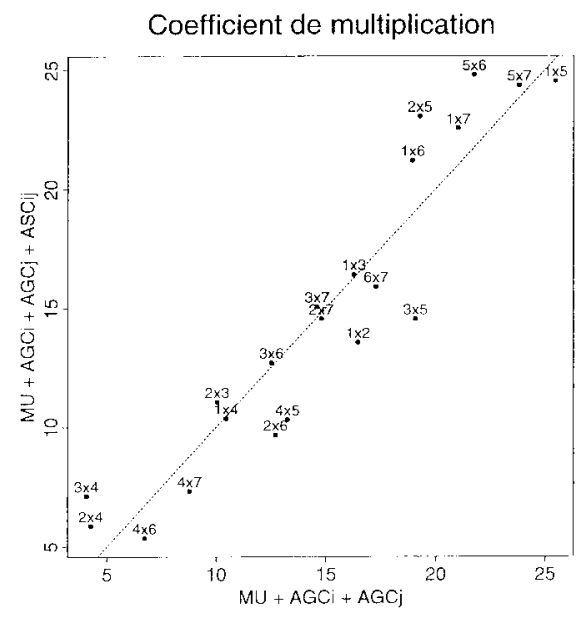

Fig 2. Liaison entre les valeurs des familles de pleins-frères prédites grâce aux AGC des parents $\left(\mu+\mathrm{AGC}_{i}+\mathrm{AGC}_{j}\right)$ et les valeurs réelles de ces familles $\left(\mu+\mathrm{AGC}_{i}+\mathrm{AGC}_{j}+\mathrm{ASC}_{i j}\right)$, pour le caractère coefficient de multiplication (coefficient de corrélation de rang $R=0,94$ ). Pour chaque point est indiqué : $n^{\circ}$ du génotype utilisé comme femelle $x n^{\circ}$ du génotype utilisé comme mâle. La ligne pointillée correspond à des effets d'ASC nuls. Les écarts verticaux des points à cette ligne correspondent aux effets d'ASC. 
Pourtant, la propagation végétative en masse, à moindre coût, de familles de pleins-frères d'élite passe non seulement par une amélioration de la technique de multiplication mais aussi, si possible, par la sélection de familles à fort coefficient de multiplication. Cette sélection n'a de sens que si elle n'est pas antagoniste d'une sélection sur les caractères d'adaptation, de vigueur ou de qualité du bois.

Pour apprécier le gain réalisable sur le coefficient de multiplication par sélection familiale, nous nous appuierons dans cet article sur une expérience de bouturage de familles de pleins-frères de douglas issues d'un plan de croisement demi-diallèle $7 \times 7$. L'intégration d'une telle sélection dans un schéma de sélection de familles sur des caractères d'intérêt sylvicole sera ensuite discutée.

\section{MATÉRIEL ET MÉTHODES}

Toutes les activités décrites dans ce qui suit ont été réalisées à la station d'amélioration des arbres forestiers du centre Inra d'Orléans. Deux expérimentations consécutives ont été conduites: l'une concerne le forçage des piedsmères, l'autre le bouturage proprement dit.

\section{Matériel végétal}

La base génétique étudiée se compose de sept génotypes de douglas vert, issus de peuplements classés français d'origine inconnue, sélectionnés phénotypiquement sur forme, vigueur, tardiveté du débourrement végétatif et densité du bois.

La figure 1 montre le plan de croisement diallèle réalisé. Afin de bénéficier d'un nombre suffisant de graines par familles, seul un demi-diallèle sans les autofécondations a été semé. Toutes les familles sont représentées par un effectif de 13 à 21 pieds-mères dans le dispositif de bouturage.

\section{Méthodes de forçage des pieds-mères}

Après un prétraitement de 5 semaines au froid humide ( 2 à $4{ }^{\circ} \mathrm{C}$ ), les graines ont été semées en janvier 1993 en capsule de tourbe de $42 \mathrm{~mm}$ de diamètre préalablement trempées dans une solution fertilisante N-P-K 20-20-20 + oligoéléments à raison de $1 \mathrm{~g} / \mathrm{L}$. Trois semaines après, les semis ont été rempotés en conteneurs de $3 \mathrm{~L}$ avec un mélange de tourbe blonde, sable et écorce de pin maritime de calibre $0-8 \mathrm{~mm}$ (4:3:3 $\mathrm{v} / \mathrm{v}$ ), dans lequel sont incorporés deux engrais à libération lente : 18-6-12 (libération 3-4 mois) à raison de $0,5 \mathrm{~kg} / \mathrm{m}^{3}$ et $15-8-11+2 \mathrm{MgO}(12-14$ mois) à raison de $3 \mathrm{~kg} / \mathrm{m}^{3}$.

Les pieds-mères ont alors été forcés sous serre en verre avec un chauffage au sol assurant au moins $18{ }^{\circ} \mathrm{C}$ dans les pots jusqu'à la fin des gelées (fin avril). La densité était de 9 plants $/ \mathrm{m}^{2}$. La température de l'air ambiant était contrôlée par des ouvrants (ouverture pour $\mathrm{T}>25^{\circ} \mathrm{C}$ ) et une ombrière aluminisée à $50 \%$ (déploiement pour $\mathrm{T}>33^{\circ} \mathrm{C}$ ). Un éclairage additionnel avec des lampes à vapeur de sodium de $400 \mathrm{~W}$ a été appliqué de 5 à 23 heures lorsque l'ensoleillement naturel était en dessous de $50 \mathrm{~W} / \mathrm{m}^{2}$. Une fertilisation à base de 15-11-15 a été apportée à chaque irrigation (goutte à goutte) de mai jusqu'à septembre. L'éclairage artificiel a été supprimé en septembre et 2 mois plus tard les piedsmères ont été placés dans une serre froide (température minimum de $2{ }^{\circ} \mathrm{C}$ ) jusqu'au prélèvement des boutures.

\section{Méthode de bouturage}

Les boutures ont été récoltées, avec un sécateur, dans la semaine du 7 au 11 février 1994. Elles mesuraient $8 \mathrm{~cm}$ et portaient toutes un bourgeon terminal. Elles ont été conservées à l'obscurité à $2{ }^{\circ} \mathrm{C}$ dans des sacs plastiques étanches jusqu'à la mise en enracinement, du 7 au 11 mars.

À l'insertion, la base des boutures a été débarrassée des aiguilles sur $1,5 \mathrm{~cm}$. Les boutures ont ensuite été immergées quelques secondes dans un bain contenant du Benlate (matière active benomyl), puis leur base a été plongée 10 secondes dans une solution aqueuse à $1000 \mathrm{ppm} / \mathrm{L}$ d'AlB . Les boutures ont été insérées dans des plaques en polystyrène surélevées de $1,5 \mathrm{~cm}$, de dimension $53 \times 30 \times 7,5 \mathrm{~cm}$, ayant 104 alvéoles de $48 \mathrm{~cm}^{3}$. Le substrat était constitué d'un mélange de $50 \%$ de tourbe blonde et de $50 \%$ de perlite (G40) préalablement humecté avec une solution aqueuse de Dériclor ( $m$ a oxyquinoléine). II ne comportait pas de fertilisation.

L'enracinement s'est effectué sous brouillard artificiel (humidité atmosphérique voisine de 
$90 \%$ pendant 1,5 mois, puis ramenée progressivement jusqu'à $70 \%$ ) en tunnel de polyéthylène double paroi (volume $266 \mathrm{~m}^{3}$ ) recouvert d'une ombrière blanche à $50 \%$. Un chauffage de fond assurait $18{ }^{\circ} \mathrm{C}$ dans le substrat jusqu'à la fin des gelées. Les portes du tunnel étaient maintenues ouvertes par très forte chaleur.

Des traitements phytosanitaires préventifs contre le botrytis ont été appliqués régulièrement en faisant alterner trois fongicides : BTF ( $\mathrm{m}$ a carbendazime, folpel, thirame), Benlate (benomyl) et Sumisclex (procymidone). Une fertilisation à base de N-P-K 20-20-20 + oligoéléments à raison de $1,5 \mathrm{~g} / \mathrm{L}$ a été appliquée à partir de la mi-juin.

Les boutures ont été extraites des alvéoles et notées entre le 18 et le 25 juillet sur la qualité de leur système racinaire.

\section{Plan d'expérience}

Le dispositif de forçage des pieds-mères était constitué de 24 blocs incomplets à composition aléatoire et le dispositif de bouturage de trois blocs complets. Les boutures prélevées sur chaque pied-mère ont été réparties de façon aléatoire et de manière équilibrée entre les trois blocs, sous forme de parcelles unitaires linéaires de taille variable (comportant 12,3 boutures en moyenne). L'identification clonale $\left(\mathrm{n}^{\circ} \mathrm{du}\right.$ piedmère) des boutures a été conservée.

\section{Mesures}

Le poids de 1000 graines de tous les lots de familles a été déterminé avant semis.

Les mesures suivantes ont été réalisées dans le dispositif de forçage:

- au cours de la saison de végétation 1993, six mesures de hauteur totale (une par mois de mai à octobre),

- en février 1994, dénombrement par pied-mère des boutures portant un bourgeon terminal (variable NB).

Dans le dispositif de bouturage, les mesures suivantes ont été prises : en juillet 1994, dénombrement, par parcelle unitaire, des boutures bien et mal enracinées (variables $\mathrm{N} 1$ et $\mathrm{N} 2$ respectivement). Ont été considérées comme mal enracinées les boutures présentant une seule racine, ou bien plusieurs racines distribuées de façon dissymétrique autour de l'axe du plant ou de longueur inférieure à $1 \mathrm{~cm}$.

Pour chaque parcelle unitaire, le taux d'enracinement (Rac) est défini par la proportion de boutures enracinées $(\mathrm{Rac}=(\mathrm{N} 1+\mathrm{N} 2) / \mathrm{NB})$ et la qualité de l'enracinement (Qual) est définie par la proportion de boutures bien enracinées parmi les boutures enracinées (Qual $=\mathrm{N} 1 /$ (N1 + N2)). Les variables NB, Rac et Qual constituent les composantes du caractère-cible " coefficient de multiplication " (Coef), c'est-à-dire nombre de boutures bien enracinées (Coef $=$ NB $\times$ Rac $\times$ Qual).

\section{Analyses statistiques}

La variabilité phénotypique a été décomposée selon les modèles additifs suivants:

Dispositif de forçage $: Y_{i j}=\mu+F_{j}+b_{j}+W_{i j}$. Dispositif de bouturage $: Y_{j k}=\mu+F_{i}+C / F_{j / i}$

$$
+b_{k}+W_{i j k}
$$

où $Y$ représente les valeurs observées ; $F$, les effets familiaux; $C / F$, les effets clonaux intra-familiaux ; $b$, les effets des blocs, et $W$, les effets résiduels. Tous les effets sont considérés comme aléatoires.

L'analyse de variance a permis de tester si les effets familiaux, clonaux et des blocs étaient significatifs (seuil choisi égal à $5 \%$ ), et d'estimer grâce à la méthode III de Henderson (Henderson, 1953) les variances familiale $\left(V_{F}\right)$, clonale intrafamiliale $\left(V_{C / F}\right)$ et résiduelle $\left(V_{W}\right)$, ainsi que les corrélations entre effets familiaux aléatoires $\left(r_{F}\right)$. Pour les couples de variables $(X, Y)$ mesurées dans des dispositifs différents, $r_{F}(X, Y)$ a été estimé par $\mathrm{r}_{\mathrm{P}}(\mathrm{X}, \mathrm{Y}) /\left[\mathrm{h}_{\mathrm{F}}(\mathrm{X}) \cdot \mathrm{h}_{\mathrm{F}}(\mathrm{Y})\right]$, où $\mathrm{r}_{\mathrm{P}}(\mathrm{X}, \mathrm{Y})$ est le coefficient de corrélation linéaire entre valeurs familiales estimées. Cette analyse de variance a porté sur les valeurs individuelles pour le dispositif de forçage et sur les valeurs parcellaires pour le dispositif de bouturage, les effectifs des parcelles étant utilisés comme pondérations.

La précision d'estimation des paramètres estimés a été appréciée par les écarts types d'échantillonnage dont les formules de calcul sont fournies par Becker (1984).

Les postulats requis pour la validité des résultats issus de l'analyse de variance ont été examinés : homogénéité des variances intrafamiliales (test de Bartlett), normalité des résidus, indépendance entre moyennes familiales et variances intra-familiales, indépendance entre valeurs estimées et résidus. Pour les variables taux de boutures enracinées et bien enracinées, les transformations de variables $\arcsin \left(x^{1 / 2}\right), \log$ $(x /(1-x))$ et $\log (x)$ entre autres ont éte essayées, dans le but de s'affranchir de la dépendance in- 
trinsèque entre moyennes et variances intra pour les variables de type proportion.

Bien que les relations d'apparentement ne soient pas prises en compte de façon explicite dans le modèle, il a été vérifié que les liaisons entre caractères au niveau familial sont similaires à celles observées au niveau parental.

Une analyse des coefficients de piste (coefficients de corrélations partieltes, Li [1975]) sur les moyennes familiales a permis de distinguer les effets directs et indirects des caractères hauteur des pieds-mères et composantes individuelles du coefficient de multiplication sur la variabilité du coefficient de multiplication. Afin de linéariser la relation Coef $=$ NB $\times$ Rac $\times$ Qual, le logarithme de ces variables a été utilisé.

\section{Espérances de gain génétique}

Pour les variables à distribution normale, l'espérance de gain génétique par sélection des meilleures familles peut s'écrire :

$$
\Delta \mathrm{G}=\mathrm{i}_{\mathrm{F}}\left[\mathrm{h}^{2} \mathrm{~F} \cdot \mathrm{V}_{\mathrm{F}}\right]^{1 / 2}
$$

avec $V_{F}$, variance entre familles estimée en modèle aléatoire ; $i_{F}$, intensité de sélection, qui dépend de la proportion de familles retenues pour une diffusion en reboisement parmi l'ensemble des familles testées; $h^{2}{ }^{2}$, héritabilité des moyennes familiales, mesurant le degré de confiance que l'on peut placer dans la moyenne phénotypique des familles pour estimer leur valeur génétique.

Selon le dispositif, ce paramètre a été estimé comme suit :

$$
\begin{gathered}
\mathrm{h}_{\mathrm{F}}^{2}=\mathrm{V}_{\mathrm{F}} /\left(\mathrm{V}_{\mathrm{F}}+\mathrm{V}_{W} / n\right) \\
\text { pour le dispositif de forçage } \\
\mathrm{h}_{\mathrm{F}}^{2}=\mathrm{V}_{\mathrm{F}} /\left(\mathrm{V}_{\mathrm{F}}+\mathrm{V}_{\mathrm{CF}} / n+\mathrm{V}_{W} / n\right) \\
\text { pour le dispositif de bouturage }
\end{gathered}
$$

avec $n$, nombre moyen de clones par famille ; $r$, nombre moyen d'observations par clone.

Pour le présent essai, $n$ et $r$ valent respectivement 19,0 et 3 .

L'intervalle de confiance de l'héritabilité des moyennes familiales a été calculé selon Knapp et al (1985).

\section{Effets de la modification de la composition clonale d'une famille}

Si une famille est évaluée précisément sous forme de semis, sa valeur génétique est modifiée, de manière imprévisible, par la propagation par bouturage en vrac. Cela tient à la variabilité du coefficient de multiplication entre piedsmères qui modifie la composition clonale de la famille. La variabilité génétique intra-familiale est également affectée.

Deux effets sont à distinguer : l'effet d'échantillonnage - un nombre limité de clones (génotypes différents) sont utilisés pour une famille issue de bouturage en vrac, et non des milliers comme pour une famille issue de semis - et l'effet de l'inégalité des contributions clonales à la composition génétique finale de la famille issue de bouturage en vrac.

Dès que le nombre de clones $n$ est suffisamment grand, l'effet d'échantillonnage a des conséquences pratiques négligeables. Ainsi, avec 100 clones par famille, s'il y a équicontribution des clones, la probabilité que la valeur génétique d'une famille $f$ soit comprise dans l'intervalle $\mu_{t} \pm 1 / 5\left[\mathrm{~V}_{\mathrm{C} / f}\right]^{1 / 2}$ est de $94 \%\left(\mu_{f}\right.$ et $V_{C / f}$ sont respectivement la moyenne familiale et la variance génétique entre clones de la famille fpour un caractère d'intérêt sylvicole).

Nous avons essayé d'apprécier l'impact pratique qu'aurait l'inégalité des contributions clonales observées dans cette expérience. Soit $p_{i}$ la proportion dans une famille de boutures bien enracinées provenant du pied-mère $i$. Si tous les pieds-mères d'une famille contribuaient de manière égale à la production de boutures, la variance d'échantillonnage de la valeur génétique moyenne de familles propagées par bouturage de $n$ pieds-mères serait : $1 / n \mathrm{~V}_{\mathrm{C} \text { /F. En pratique, }}$ les $n$ pieds-mères d'une famille contribuent en proportion $p_{1}, p_{2}, \ldots, p_{i}, \ldots, p_{n}$ à sa composition génétique. En conséquence, la variance d'échantillonnage de la valeur génétique moyenne de familles propagées par bouturage de $n$ piedsmères ayant des contributions clonales données $p_{1}, \ldots, p_{n}$ vaut :

$$
\left(p_{1}{ }^{2}+p_{2}{ }^{2}+\ldots+p^{2}+\ldots+p_{n}{ }^{2}\right) V_{C / F}>1 / n V_{C / F}
$$

cela en supposant une indépendance de ces contributions relatives avec les valeurs génétiques clonales du caractère d'intérêt sylvicole considéré.

Le rapport $\mathrm{D}=\left[\left(p_{1}^{2}+\ldots+p_{n}{ }^{2}\right) /(1 / n)\right]^{1 / 2}$ nous servira pour apprécier l'effet d'inégalité des contributions clonales d'une famille.

\section{Effets d'AGC et d'ASC}

Grâce au plan de croisement diallèle, la variabilité génétique familiale peut être décomposée en parts dues aux effets d'aptitude générale et spécifique à la combinaison (AGC et ASC). Ces effets ont été considérés comme fixés. 
Tableau I. Variabilité familiale, héritabilité familiale et importance relative des effets d'ASC par rapport aux effets d'AGC pour les caractères mesurés.

Composantes du taux de multiplication

\begin{tabular}{|c|c|c|c|c|c|}
\hline & \multirow[b]{2}{*}{$H T$} & & \multirow[b]{2}{*}{ Coef } \\
\hline & & NB & $R a c$ & Qual & \\
\hline Moyenne & $56 \mathrm{~cm}$ & 36 & $72 \%$ & $49 \%$ & 15 \\
\hline Valeur familiale minimum & $49 \mathrm{~cm}$ & 26 & $48 \%$ & $27 \%$ & 6 \\
\hline Valeur familiale maximum & $63 \mathrm{~cm}$ & 45 & $92 \%$ & $73 \%$ & 26 \\
\hline $\begin{array}{l}\text { Coefficient de variation familial } \\
\text { Parts dans la variance }\end{array}$ & $5,3 \%$ & $13 \%$ & $16 \%$ & $24 \%$ & $42 \%$ \\
\hline \multicolumn{6}{|l|}{ Phénotypique des variances : } \\
\hline familiale & $11 \%$ & $25 \%$ & $3,6 \%$ & $2,7 \%$ & $6,0 \%$ \\
\hline clone/famille & - & - & $2,5 \%$ & $4,4 \%$ & $7,0 \%$ \\
\hline résiduelle & $89 \%$ & $75 \%$ & $91,2 \%$ & $92,9 \%$ & $87,0 \%$ \\
\hline Héritabilité familiale & 0,71 & 0,87 & 0,90 & 0,88 & 0,92 \\
\hline [Intervalle de confiance à $95 \%$ ] & {$[0,49-0,86]$} & {$[0,77-0,94]$} & {$[0,83-0,95]$} & {$[0,79-0,94]$} & {$[0,87-0,96]$} \\
\hline$R$ & 0,87 & 0,94 & 0,95 & 0,94 & 0,94 \\
\hline
\end{tabular}

$R$ : coefficient de corrélation de rang entre les valeurs des familles de pleins-frères et leurs valeurs estimées par la somme des AGC parentales. HT, hauteur finale des semis de 1 an ; NB, nombre de boutures prélevables par semis; Rac, taux de boutures enracinées; Qual, taux de boutures bien enracinées parmi les boutures enracinées ; Coef, coefficient de multiplication.

Le modèle utilisé est :

$$
\mathrm{Y}_{i j k}=\mu+\mathrm{AGC}_{i}+\mathrm{AGC}_{j}+\mathrm{ASC}_{i j}+\mathrm{W}_{i j k}
$$

où $A G C_{i}, A G C_{i}$ et $A S C_{i j}$ sont les effets d'AGC et d'ASC des parents $i$ et $j$. L'analyse de variance a permis de tester la signification des effets d'AGC et d'ASC. L'importance des effets d'ASC par rapport à ceux d'AGC a été appréciée par la corrélation de rang, $R$, entre les valeurs des familles de pleins-frères: $\mu+A G C_{j}+A G C_{j}+A S C_{i j}$ et les valeurs de ces familles prédites grâce à l'AGC des parents : $\mu+\mathrm{AGC}_{i}+\mathrm{AGC}_{\text {. }}$.

Aucune hypothèse n'est faite sur le mode d'action des gènes, à part celle d'absence d'effets réciproques. Les calculs ont été réalisés sur les données ajustées des effets des blocs avec le programme dial du logiciel Opep (Baradat, 1989).

\section{RÉSULTATS}

En fin de période de forçage, la hauteur totale à un an des pieds-mères sur lesquels les boutures ont été prélevées (HT) était en moyenne de $56 \mathrm{~cm}$. Sur l'ensemble de l'expérience, en moyenne, le nombre de boutures terminales prélevables par pied-mère (NB) était de 36 , le taux de boutures enra- cinées (Rac) était de $72 \%$ et de boutures bien enracinées parmi les boutures enracinées (Qual) de $49 \%$. Finalement, le coefficient de multiplication (Coef) était en moyenne de 15 boutures bien enracinées par pied-mère.

La hauteur finale à un an résume toute l'information, au niveau des familles, sur les hauteurs successives mesurées sur les pieds-mères. En effet, la courbe moyenne de croissance en hauteur de chaque famille est linéaire et il existe une liaison presque totale entre moyennes familiales pour le taux d'accroissement et pour la hauteur finale.

La hauteur finale est le seul caractère pour lequel l'effet des blocs est significatif (au seuil de $5 \%$ ). Pour les autres caractères, la dispersion entre valeurs estimées des blocs est très faible par rapport à la dispersion entre valeurs familiales.

Tous les caractères présentent à la fois une forte variabilité et une forte héritabilité familiales (tableau I). Ainsi, le nombre de boutures prélevables et le taux d'enracine- 
Tableau II. Valeurs estimées des corrélations interfamiliales pour la hauteur des pieds-mères, les composantes du coefficient de multiplication et le coefficient de multiplication.

\begin{tabular}{lrrrrrr}
\hline & $H T$ & NB & Rac & Qual & Coef & D \\
\hline HT & 1 & & & & & \\
NB & 0,52 & 1 & & & & \\
Rac & $-0,05$ & 0,58 & 1 & & & \\
Qual & 0,01 & 0,56 & 0,81 & 1 & & \\
Coef & 0,16 & 0,77 & 0,90 & 0,92 & 1 & 1 \\
$D^{\text {a }}$ & $-0,09$ & $-0,65$ & $-0,86$ & $-0,84$ & $-0,78$ & \\
\hline
\end{tabular}

HT, hauteur finale des semis de 1 an ; NB, nombre de boutures prélevables par semis; Rac, taux de boutures enracinées : Qual, taux de boutures bien enracinées parmi les boutures enracinées ; Coef, coefficient de multiplication ; D, paramètre mesurant le degré de modification de la composition clonale d'une famille après multipication

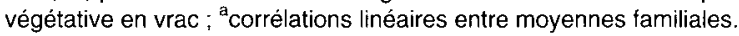

ment varient pratiquement du simple au double entre familles extrêmes et le coefficient de multiplication dans un rapport de 1 à 4. On note que l'héritabilité du nombre de boutures prélevables (NB), lié à l'architecture du pied-mère, est bien plus forte que celle de la hauteur. Bien qu'on ne puisse pas en toute rigueur comparer la variabilité des caractères mesurées dans les dispositifs de forçage et de bouturage, la part de la variance génétique dans la variance totale apparaît plus forte pour NB que pour les caractères liés à l'enracinement (25 contre 6-7\%).

Au niveau des moyennes de familles, les composantes du coefficient de multiplication peuvent être liées favorablement ou très favorablement entre elles $\left(0,6<r_{F}<0,9\right)$. La hauteur des plants n'est liée qu'au nombre de boutures prélevables

Tableau III. Coefficients de piste sur les moyennes familiales pour distinguer les effets directs et indirects des caractères hauteur des pieds-mères et composantes individuelles du coefficient de multiplication sur la variabilité du coefficient de multiplication.

\begin{tabular}{lccccc}
\hline & \multicolumn{3}{c}{ Composantes du taux de multiplication } \\
\cline { 5 - 6 } & $H T$ & $\log (\mathrm{NB})$ & $\log ($ Rac $)$ & $\log ($ Qual) & $\begin{array}{c}\text { Part non } \\
\text { expliquée }\end{array}$ \\
\hline Effets directs & $-0,02$ & 0,31 & 0,20 & 0,61 & 0,03 \\
& & & & & \\
Effets indirects via & - & $-0,01$ & 0,00 & 0,00 \\
HT & 0,13 & - & 0,16 & 0,16 \\
$\log$ (NB) & $-0,01$ & 0,11 & - & 0,19 \\
$\log$ (Rac) & $-0,03$ & 0,31 & 0,57 & - \\
$\log$ (Qual) & 0,09 & 0,72 & 0,94 & 0,96 \\
Total = coefficients de & & & &
\end{tabular}


(NB) (tableau II). Toutefois, en utilisant la hauteur totale des pieds-mères comme covariable, la variance familiale pour NB n'est diminuée que de $15 \%$.

Aucune des variables n'est liée au poids moyen des lots de graines des familles (les corrélations linéaires entre moyennes familiales sont très faibles et non significatives au seuil de $5 \%$ ).

L'analyse des coefficients de piste présentée au tableau III montre que la corrélation entre moyennes familiales des caractères NB et Coef n'est pas due à un effet indirect de la hauteur totale des piedsmères, auquel NB est lié, mais en revanche est en grande partie expliquée par les variables d'enracinement, et que c'est le caractère de qualité d'enracinement qui contribue le plus à la variabilité entre familles du coefficient de multiplication.

Les distributions des moyennes familiales de toutes les variables étudiées ont une allure normale, ce qui permet d'estimer les gains génétiques espérés par sélection des meilleures familles de pleins-frères pour l'aptitude au bouturage. Ainsi, dans cet essai, un taux de sélection des familles $p=10 \%$ ferait passer le coefficient de multiplication de 15 à 26 , soit une augmentation très sensible $(+73 \%)$ du rendement de la technique de multiplication végétative.

Il existe par ailleurs une forte corrélation négative, favorable, entre coefficient de multiplication et modification de la composition clonale des familles. La liaison est encore plus favorable pour les variables de taux et de qualité de l'enracinement (tableau II). Le paramètre $D$, mesurant l'impact pratique de la modification de la composition clonale sur la "dérive " de la valeur génétique familiale, estimée sous forme de semis, est très proche de 1 (impact faible) pour les familles présentant un taux de multiplication supérieur à 20 .

Les effets d'ASC sont significatifs au seuil de $5 \%$ pour tous les caractères, exceptés ceux mesurés dans le dispositif de forçage, mais dans tous les cas les effets d'ASC sont faibles devant les effets d'AGC. En effet, les corrélation de rang, $R$, entre les valeurs des familles de pleins-frères et les valeurs de ces familles prédites grâce à l'AGC des parents sont très fortes (tableau l et fig 2).

\section{DISCUSSION}

\section{Validité et généralité des résultats obtenus}

Les résultats obtenus ne seraient a priori extrapolables qu'aux seules conditions d'élevage et de bouturage similaires à celles de l'expérience, ainsi que pour les sept parents du plan diallèle. Une deuxième expérience de bouturage sur le même matériel, cette fois-ci avec des conditions de forçage plus rustiques, moins coûteuses, permettra d'étudier la stabilité du classement des familles, essentielle pour une application pratique des résultats trouvés dans la présente étude. Par ailleurs, pour limiter la taille de l'expérimentation, seules les boutures présentant des bourgeons terminaux ont été récoltées. Une expérience réalisée en 1992 à l'Inra d'Orléans sur 84 clones de douglas a montré une bonne concordance pour les taux d'enracinement des boutures terminales et intermédiaires (coefficient de corrélation de rang au niveau clonal égal à $0,68, p<0,001)$; il n'y avait pas de différences significatives de croissance, survie et plagiotropie entre boutures terminales et intermédiaires (Verger, non publié). Les coefficients de multiplication de l'ordre de 25 obtenus dans la présente expérience pour les meilleures familles seraient au moins doublés par la récolte des boutures intermédiaires en plus des boutures terminales. Cela permettrait d'atteindre des chiffres de l'ordre de 50 obtenus par la compagnie Weyerhaeuser pour la production en masse de boutures de familles de pleinsfrères d'élite de douglas (Ritchie, 1992).

Pour les variables Rac et Qual, de type proportion, qui présentent une forte liaison entre moyennes familiales et variances in- 
trafamiliales, aucune transformation de variable satisfaisante n'a été trouvée. Cependant, les espérances de gains génétiques calculés pour un taux de sélection de $10 \%$ sont dans tous les cas proches de la moyenne des deux meilleures parmi les 21 valeurs familiales estimées, représentant un taux de sélection comparable. Ces valeurs familiales estimées devraient être proches des valeurs génétiques vraies du fait des fortes héritabilités des moyennes familiales.

Les effets liés à l'environnement des pieds-mères et à l'histoire des boutures (dits effets « $C$ ») peuvent être une source importante de biais pour l'estimation des paramètres génétiques (Radosta et al, 1994). Ils peuvent aussi contribuer à l'interaction génotype-milieu (conditions de forçage et de bouturage, année). Dans cette expérience, ils ont toutefois été réduits le plus possible. En effet, les pieds-mères sur lesquels les boutures ont été prélevées étaient situés dans un environnement aussi homogène que possible et de plus étaient randomisés dans le dispositif de forçage. En outre, le prélèvement de boutures sur les pieds-mères, qui avaient tous le même âge et étaient au même stade physiologique, a été réalisé de manière aussi homogène que possible. Différents auteurs (Foster et al, 1984 ; Farmer et al, 1989) ont ainsi trouvé que les effets $C$ contribuaient peu à la variation génétique totale lorsque les pieds-mères ont peu eu le temps de développer des aptitudes au clonage différentielles.

Enfin, les fortes liaisons positives observées entre les composantes du coefficient de multiplication, au niveau familial, sont également observées au niveau des génotypes parents des familles. De même, l'absence de liaison entre la hauteur finale des semis et les variables d'enracinement se retrouve au niveau parental. Ces observations permettent de penser que les liaisons entre caractères au niveau familial ne sont pas biaisées par les relations d'apparentement entre les familles du diallèle.

\section{Sélection de familles de pleins-frères à fort coefficient de multiplication}

Le coefficient de multiplication dépend successivement :

- du nombre de boutures prélevables par pied-mère,

- de l'enracinement de ces boutures.

En ce qui concerne la première composante, une variabilité importante pour le nombre de branches (égal au nombre de boutures terminales prélevables) a été observée par Coulaud (1989) et Héois (1994) entre familles de demi-frères de douglas sur des semis de 2 ans non forcés. Dans ces essais, le nombre de branches est fortement corrélé à la hauteur totale des semis. Cette liaison est favorable dans le sens où une sélection pour la vigueur juvénile tendra à augmenter le nombre de boutures prélevables, mais serait défavorable si le nombre de branches à 1 an est lié à une dépréciation de la qualité future du bois ou si la vigueur juvénile est liée négativement avec la vigueur adulte. Dans le présent essai, une sélection sur la hauteur des pieds-mères conserverait une variabilité importante pour le nombre de boutures prélevables.

En ce qui concerne l'aptitude à l'enracinement adventif de boutures, Haissig et Riemenschneider (1988) notent qu'il existe peu de connaissances sur ses manifestations biochimiques et physiologiques. Cependant, une variabilité significative entre familles de pleins-frères d'espèces d'arbres forestiers pour l'aptitude à l'enracinement a été mise en évidence dans plusieurs études (Skrøppa et Dietrichson (1986) sur épicéa commun, Foster (1990) sur Pinus taeda, Verger et Pâques (1993), Radosta et al (1994) sur Larix x eurolepis et Borralho et Wilson (1994) sur Eucalyptus globulus). La proportion de la variance génétique totale dans la variance phénotypique est de l'ordre de 0,4 pour l'expérience de Skrøppa et Dietrichson, alors qu'elle est de l'ordre de 0,1 pour les expériences de 
Foster et de Radosta et al, comme dans le présent essai. Alazard (1992), pour des semis de pin maritime de 6 mois à excellente aptitude à l'enracinement, n'a pas trouvé de différences significatives entre familles. La très forte liaison entre taux et qualité d'enracinement des boutures dans cette expérience laisse à penser qu'il s'agit d'un seul et même caractère, à seuils, d' " aptitude à l'enracinement ". II existe des techniques statistiques adaptées au cas de variables normales sous-jacentes à des caractères à seuils (Foulley et Manfredi, 1991). Par ailleurs, l'absence de liaison entre l'aptitude à l'enracinement et la hauteur des plants dans les conditions de notre expérience semble indiquer que la variation génétique observée provient essentiellement de gènes impliqués directement dans l'enracinement.

Dans cet essai, il existe une liaison modérée entre le nombre de branches et l'aptitude à l'enracinement. Une explication possible est que la propension à faire de nombreuses branches et à avoir une aptitude à l'enracinement élevée traduisent une forte juvénilité.

Même en l'absence de liaison entre NB et aptitude à l'enracinement des boutures, on s'attend à ce que la variabilité de NB contribue de façon importante à celle du coefficient de multiplication (Coef), dont il est une composante. La corrélation positive entre NB et Coef permet d'alléger les mesures d'enracinement des boutures en éliminant dans une première phase de sélection les plus mauvaises familles pour NB. Par exemple, pour un taux de sélection final de $10 \%$, en supposant que NB est aussi héritable que Coef et que la corrélation au niveau familial entre ces deux caractères est de 0,3 , une élimination de $10 \%$ des familles sur NB dans une première étape conduira à une perte de seulement $1 \%$ sur le gain génétique attendu sur Coef (méthode : Cotterill et James, 1981).

Pour le coefficient de multiplication, la très forte liaison entre les valeurs des familles de pleins-frères et leurs valeurs prédites par la somme des AGC parentales (fig 2) rendrait très efficace une sélection sur AGC des parents potentiels des meilleures familles. Si ces résultats sont confirmés, il ne serait pas nécessaire de tester les familles obtenues par croisements des parents présentant les meilleures AGC pour le coefficient de multiplication.

La relation favorable trouvée dans cette expérience entre taux de boutures bien enracinées et faible modification de la composition clonale doit être générale. En effet, plus une famille a un taux d'enracinement proche de $100 \%$ et plus les clones de la famille auront des taux d'enracinement, donc des coefficients de multiplication, proches entre eux. En pratique, dans une pépinière de production, le nombre de clones bouturés par famille utilisé serait très supérieur à la vingtaine retenus dans cet essai. Il serait de l'ordre de la centaine au moins. La variance d'échantillonnage de la valeur génétique moyenne des familles sera donc suffisamment faible pour ne pas avoir d'impact pratique tangible, même si l'on tient compte de l'accroissement de cette variance par rapport au cas équilibré dû à l'inégalité des contributions clonales. À coefficient de multiplication égal, vaut-il mieux avoir des familles à fort taux de boutures enracinées de bonne qualité que des familles à fort nombre de boutures prélevables ? La comparaison des coûts impliqués par les phases d'élevage des pieds-mères et de mise en enracinement permettrait de répondre à cette question.

Quel serait l'effectif en pieds-mères et boutures par pieds-mères pour estimer avec suffisamment de précision, dans un objectif de sélection, le coefficient de multiplication de familles? Les résultats de cet essai et l'expérience acquise sur les tests de familles de pleins-frères (Cotterill et James, 1984) montrent que 20 clones par famille seraient suffisants. Par ailleurs, comme l'on est intéressé par l'évaluation 
Tableau IV. Proposition d'intégration de la sélection de familles à fort coefficient de multiplication dans un schéma de sélection de familles de pleins-frères pour des caractères sylvicoles.

\begin{tabular}{|c|c|c|c|}
\hline$\hat{A g g e}$ & $\begin{array}{l}\text { i) Sélection de familles FS } \\
\text { sur caractères sy/vicoles } \\
\text { ii) Semis des familles FS retenues } \\
\text { et sélection finale sur Coef }\end{array}$ & $\begin{array}{l}\text { i) Sélection de familles FS } \\
\text { sur Coef } \\
\text { ii) Test en forêt (sous forme de } \\
\text { boutures) des familles FS retenues }\end{array}$ & $\begin{array}{l}\text { i) Sélection sur Coef des parents } \\
\text { potentiels des variétés FS } \\
\text { ii) Test en forêt des familles FS } \\
\text { créées à partir des meilleurs parents }\end{array}$ \\
\hline 1 & Croisements FS & Croisement FS & Croisement HS \\
\hline 2 & Semis & Semis et élevage des pieds-mères & Semis et élevage des pieds-mères \\
\hline 3 & & Bouturage.Sélection sur Coef & Bouturage. Sélection sur Coef \\
\hline 4 & Plantation & & Croisements FS \\
\hline 5 & & & Semis \\
\hline 6 & & Plantation & | \\
\hline 7 & & & Plantation \\
\hline 8 & & & \\
\hline 9 & & & \\
\hline 10 & Sélection en forêt & & \\
\hline 11 & Semis et élevage des pieds-mères & $\downarrow$ & \\
\hline 13 & Bouturage. Sélection sur Coef & Sélection en forêt & $\downarrow$ \\
\hline 14 & & & Sélection en forèt \\
\hline
\end{tabular}

On suppose ici que la sélection en forêt est effectuée après 8 ans de plantation. Coef, coefficient de multiplication ; FS, croisements contrôlés, donnant lieu à des familles de plein-frères (full-sibs); HS, croisements par pollinisation naturelle ou mélange pollinique, donnant lieu à des familles de type demi-frères (familles half-sibs).

précise des valeurs familiales et non clonales, une dizaine de boutures par piedsmères devraient suffire pour évaluer précisément l'aptitude à l'enracinement de ces pieds-mères.

\section{Intégration de la sélection de familles à fort coefficient de multiplication dans un schéma de sélection pour des caractères sylvicoles de familles de pleins-frères}

Trois schémas sont envisageables (tableau IV) :

i) Une sélection des familles de pleinsfrères sur des critères d'intérêt sylvicole, suivie d'une sélection sur leur aptitude au bouturage (cas général).

Cette solution est adaptée pour les cas où une information sur la valeur sylvicole des familles est déjà disponible. La sélection pour l'aptitude au bouturage ajoute une étape d'une durée de 2 ans, relativement faible par rapport au temps de sélection en milieu forestier.

ii) Une évaluation des familles de pleinsfrères sur aptitude au bouturage puis critères sylvicoles.

Cette solution consiste à tester en forêt des familles préalablement multipliées par voie végétative, avec élimination avant plantation des familles à faible taux de multiplication. Le matériel testé, issu de boutures et non de semis, correspond alors au matériel destiné à être diffusé pour le reboisement. Ceci permet d'intégrer d'éventuels effets liés au bouturage. Cette solution se justifie en fait surtout si elle est couplée à une sélection individuelle sur valeur clonale dans les familles dans le cadre d'un schéma de sélection récurrente (exemple Karlsson et Rosval [1993] sur l'épicéa commun en Suède).

iii) Une évaluation préalable de l'AGC des parents pour l'aptitude au bouturage (par 
des croisements par pollinisation naturelle ou par des mélanges polliniques), avant le test en forêt.

Cette solution présente plusieurs avantages : pouvoir effectuer une forte intensité de sélection sur l'aptitude au bouturage, puisqu'on peut tester beaucoup plus de parents que de familles de pleins-frères; pouvoir créer, à partir d'un ensemble de parents donné, les familles qui auront a priori le plus fort coefficient de multiplication ; réduire le nombre de familles à tester en forêt à celles susceptibles de bien se multiplier végétativement. L'évaluation des parents pourrait également s'effectuer en pépinière sur d'autres caractères d'intérêt sylvicole, qui doivent présenter une forte corrélation juvénile-adulte et des effets d'ASC faibles devant ceux d'AGC.

L'efficacité de ce schéma repose sur la généralité des résultats trouvés dans cette expérience sur la prédominance des effets d'AGC pour les variables liées à l'aptitude au bouturage. Par ailleurs, si l'aptitude au bouturage est liée à une mauvaise croissance ou ramification au stade adulte, le danger d'une contre-sélection pour des caractères d'intérêt sylvicole existe.

\section{CONCLUSION}

L'utilisation de variétés à base génétique plus étroite que les vergers à graines, mais possédant néanmoins une variabilité génétique non négligeable, semble présenter de nombreux atouts : gain génétique important, produits plus homogènes que ceux issus de variétés de types populations ou synthétiques, flexibilité de la création variétale pour renouveler les variétés (variétés plus performantes ou spécialisées).

De nombreux problèmes restent néanmoins à résoudre avant la diffusion de telles variétés:

- Avant tout des problèmes technico-économiques : le prix de vente du plant par rapport au plant issu de semis des variétés classiques ne doit pas être trop élevé afin de ne pas décourager les reboiseurs, et d'autre part la diffusion des résultats sur la supériorité du matériel créé et la mise en place de tests de démonstration sont nécessaires.

- Des problèmes génétiques et sylvicoles: le supplément théorique de gain génétique apporté par les variétés pleins-frères peut être surestimé si la réduction de la variabilité génétique présente dans un peuplement restreint la possibilité de sélection sylvicole par éclaircie, pour faire face à l'hétérogénéité des sites de reboisements dans l'espace et le temps. Les itinéraires sylvicoles, valorisant au mieux ce type de matériel fortement amélioré génétiquement, sont à mettre au point : nombre optimum de familles à inclure dans un reboisement, mélange pied à pied ou en blocs des familles, espacement optimal et utilisation éventuelle de plants tout venant, moins chers, destinés à être enlevés en éclaircie précocement.

Cette expérience a démontré qu'il existe un fort potentiel de sélection pour le coefficient de multiplication de familles de pleinsfrères de douglas. L'amélioration des techniques de multiplication végétative peut rendre inutile une étape de sélection pour le coefficient de multiplication. Cependant, il est aussi possible d'envisager d'utiliser des techniques de forçage rustiques, moins coûteuses en intrants, et de compenser la diminution éventuelle de succès du bouturage par rapport aux techniques plus sophistiquées par la sélection pour l'aptitude au bouturage. Cette voie est en cours d'étude à l'Inra et au Cemagref.

L'essai dont il a été rendu compte ici a été transféré sur le terrain pour évaluer sur le long terme le comportement des boutures par rapport à des témoins issus de semis.

\section{REMERCIEMENTS}

Cette étude a été financée par les contrats GIS MRT " Bulk douglas " et CEE (DG XII) " Conifères à croissance rapide " (contrat AIR 
$\left.n{ }^{\circ} \mathrm{CT} 920143\right)$. Les opérations techniques ont été menées à bien, à des titres divers, grâce aux personnes suivantes: MM A Perru, D Piermant, H Miteul, G Sapin, D Plazanet et E Popov; $M^{\text {mes }}$ $F$ Boyé et I Len ; Melles $S$ Bay, $V$ Godet et M Millard; qu'elles en soient à nouveau remerciées. Nous remercions également C Bastien (InraOrléans) pour les discussions constructives que nous avons eues avec elle.

\section{RÉFÉRENCES}

Alazard P (1992) Aspects techniques et économiques de la multiplication de masse chez le pin maritime. In : Production de variétés génétiquement améliorées d'espèces forestières à croissance rapide. Symposium Afocel-lufro, Bordeaux, 1992, tome I, 365-373

Baradat $P$ (1989) Amélioration génétique des arbres forestiers. Éléments méthodologiques. Document interne Inra

Becker WA (1984) Manual of quantitative genetics. Academic Enterprises, Pullman, WA, Etats-Unis, $188 \mathrm{p}$

Borralho NMG, Wilson PJ (1992) Inheritance of initial survival and rooting ability in Eucalyptus globulus Labill stem cuttings. Silvae Genet 43, 238-242

Cotterill PP, James JW (1981) Optimising two-stage independent culling selection in tree and animal breeding. Theor Appl Genet 59, 67-72

Cotterill PP, James JW (1984) Number of offspring and plot size required for progeny testing. Silvae Genet 33. 203-209

Coulaud S (1989) Mise au point de tests de sélection précoce chez le douglas. Mémoire de fin d'ètude de I'ENITHP

Farmer RE, Freitag M, Garlick K (1989) Genetic variance and $C$ effects in balsam poplar rooting. Sivae Genet 38, 62-65

Fletcher AM (1992) Breeding improved Sitka spruce. In : Super Sitka for the 90s. Forestry Commission Butletin 103, 11-24

Foster GS (1990) Genetic control of rooting ability of stem cuttings from loblolly pine. Can J For Res 20, 1361-1368

Foster GS, Campbell RK, Adams WT (1984) Heritability, gain and $C$ effects in rooting of stem western hemlock cuttings. Can J For Res 14, 628-638

Foulley JL, Manfredi E (1991) Approches statistiques de l'évaluation génétique de reproducteurs pour des acaractères binaires à seuil. Genet Sel Evol 23, 309-338

Haissig BE, Riemenschneider DE (1988) Genetic effects on adventitious rooting. In : Adventitious Roof Formation in Cuttings (TD Davies, BE Haissig, $N$ Sankhla, eds, ), Advances in Plant Sciences Series, Dioscorides Press, Portland, OR, volume 2, 47-60

Henderson CR (1983) Estimation of variance and covariance components. Biometrics 9, 226-252
Héois $B$ (1994) Variabilité génétique chez Pseudotsuga menziesii (Mirb) Franco. Contribution à la mise au point de tests précoces. Thèse Ina-PG

Karlsson B, Rosvall O (1993) Bredding programmes in Sweden-Norway spruce. Proc-Nordic Group for Tree Breeding. Edinburgh, UK, 128-134

Kleinschmit J (1979) Limitations for restriction of the genetic variation. Silvae Genet 28, 61-67

Knapp SJ, Stroup WW, Ross WM (1989) Exact confidence intervals for heritability on a progeny mean basis. Crop Sci 25, 192-194

Li CC (1975) Path analysis - a primer. The Boxwood Press, Pacific Grove, CA, Ėtats-Unis

Libby WJ (1990) Advantages of Clonal Forestry Revisited. Proc of the Joint Meeting of Western Forest Genetics Association and IUFRO Working Parties. Olympia, WA, États-Unis, 5.18

Michaud D, Gautry JY, Bouvet A (1990) First results in plantation of Douglas-fir clones. Proc of the Joint Meeting of Western Forest Genetics Association and $U$ FRO Working Parties. Olympia, WA, États-Unis, 5.31

Radosta P, Pâques LE, Verger M (1994) Estimation of genetic and non-genetic parameters for rooting traits in hybrid larch. Silvae Genet 43, 108-114

Ritchie GA (1992) Commercial production of Douglas-fir rooted cuttings at Weyerhaeuser Company. In : Production de variétés génétiquement améliorées d'espèces forestières à croissance rapide. Symposium Afocel-Iufro, Bordeaux 1992, tome II, 363-370

Ritchie GA (1993) Production of Douglas-fir, Pseudotsuga menziesii (Mirb) Franco, rooted cuttings for reforestation by Weyerhaeuser Company. In : Combined Proceedings International Plant Propagator's society, volume $43,284-288$

Schermann N (1994) Étude des paramètres génétiques de trois populations de douglas vert (Pseudotsuga Menziesii (Mirb) Franco). Analyse d'un diallele $16 \mathrm{x}$ 16. Conséquences pour la stratégie d'amélioration génétique de l'espèce. Thèse de docteur-ingénieur de l'Ina-PG, $117 p$

Shelbourne CJA (1986) The role of cuttings in the genetic improvement of forest trees. In : Workshop on Growing Radiata Pine from Cuttings. Rotorua 5-7 mai 1986. New Zealand Forest Research Institute Bulletin 135, 7-17

Skrøppa T, Dietrichson J (1986) Genetic variation and ortet/ramet relationships in a clonal test with Picea abies. Scand J For Res 1, 323-332

Talbert CB, Ritchie GA, Gupta P (1993) Conifer vegetative propagation: an overview from a commercialisation perspective. In : Clonal Forestry I, Genetics and Biotechnology (MR Ahuja, WJ Libby, eds), Springer-Verlag, Berlin 145-181

Verger M, Pâques LE (1993) Multiplication végétative du mélèze hybride (Larix $x$ eurolepis) par bouturage en vrac. Ann. Sci. For. 50, 205-215 